\title{
A site-directed chromosomal translocation induced in embryonic stem cells by Cre-IoxP recombination
}

Andrew J.H. Smith, Michelle A. De Sousa, Bernard Kwabi-Addo, Amanda Heppell-Parton, Helen Impey \& Pamela Rabbitts

Nature Genetics 9, 376-385 (1995)

In the legend to Fig. 7, the descriptions of the recombinant chromosomes indicated by the arrows were inadvertantly reversed and the legend should have read 'the long arrow identifies the recombinant chromosome 15 containing the translocated telomeric region of 12 and the short arrow the recombinant chromosome 12 containing the translocated telomeric region of $15^{\prime}$. Our error does not affect our conclusions in any way.

\section{Epileptic seizures caused by inactivation of a novel gene, jerky, related to centromere binding protein-B in transgenic mice}

Miklos Toth, Joseph Grimsby, Gyorgy Buzsaki \& Gerald P. Donovan

Nature Genetics 11, 71-75 (1995)

Upon analysis with an ABI 373 automated sequencer, we have discovered one additional base in the published jerky gene sequence that appeared compressed using manual sequencing techniques. This base causes a frameshift near the end of the open reading frame, resulting in a putative $58 \mathrm{kD}$ gene product rather than the $41.7 \mathrm{kD}$ protein predicted in the paper. This change does not affect the published homologies at the $\mathrm{N}$ terminus of the jerky protein. The corrected sequence has been deposited in Genbank (Accession number U35730).

\section{The carrier frequency of the BRCA1 185delAG mutation is approximately 1 percent in Ashkenazi Jewish individuals}

Jeffrey P. Struewing, Dvorah Abeliovich, Tamar Peretz, Naaman Avishai, Michael M. Kaback, Francis S. Collins \& Lawrence C. Brody

Nature Genetics 11, 198-200 (1995)

The sequence of the 185delAG mutant oligonucleotide described in the Methods section contained an error. The correct sequence should read: $5^{\prime}-$ atc tta gtg tce cac tc- $3^{3}$. Hybridization with either this oligonucleotide, or the sequence originally reported in Struewing et al., yield comparable results and do not alter the conclusions of the study. The authors regret this error.

\section{$\beta$-sarcoglycan (A3b) mutations cause autosomal recessive muscular dystrophy with loss of the sarcoglycan complex}

Carsten G. Bönneman, Raju Modi, Satoru Noguchi, Yuji Mizuno, Mikiharu Yoshida, Emanuela Gussoni, Elizabeth N. McNalley, David J. Duggan, Corrado Angelini, Eric P. Hoffman, Eijiro Ozawa \& Louis M. Kunkel

Nature Genetics 11, 266-273 (1995)

i) Under "Patient screening and identification of mutations", the sixth sentence in the second paragraph should read: The second conformer had an 8 base pair duplication after position 125, which causes a frameshift and an immediate stop codon after position 129 (Fig. 4b).

ii) In figure $4 b$, the numbering was off by one:

B\&D

Normal ${ }_{179}^{535}$ TTA TTC AGC $\ldots$ 